\title{
Preface: Genomic Pathology and Cancer Biomarkers: Toward Precision Medicine
}

Major advances of genomic technologies have greatly expanded the repertoire of cancer biomarkers, guiding the practice of precision medicine. In this special issue, we have invited a group of experts to provide a comprehensive review on the application of genomic pathology and the use of a wide spectrum of cancer biomarkers.

First, prostate cancer biomarkers and their molecular bases are reviewed in two important contributions by Hodges et al. and Iczkowski. Emphasis is placed on their importance in the diagnosis and prognostication of prostate cancer. $\mathrm{Lu}$ and colleagues focused their contribution on the emerging concepts and methodologies in cancer biomarker's discovery, which is a critical part of cancer prevention and treatment. The types of liquid biopsies currently available and their potential clinical applications involved in bladder cancer are discussed in detail by Yang and colleagues. $\mathrm{Ni}$ et al. provide an overview of gene fusions in various types of tumors, covering diagnostic biomarkers, method selections, and treatments.

Remarkable advances in the molecular and genomic studies of breast cancer are reviewed by Zhang and Tang. The treatment of triple-negative breast cancer based on biomarkers of DNA repair and related pathways is presented by Guo and colleagues. Although researchers have made great strides in the treatment of some types of breast cancer, the treatment of triple-negative breast cancer is yet to be fully elucidated. This contribution is followed by a review of epigenetic modifications in prostate cancer by Cimadamore and Montironi. Polyploid giant cancer cells, stem-like "antaeus" in cancer, are discussed by Chen et al. Fang and colleagues summarize the immunohistochemical biomarkers that can be used to reflect on the molecular pathology, and they focus on their applications in the pathology diagnosis and differential diagnosis. Luchini et al. focused their attention on the SWI/SNF complexes and three of the most important chromatin remodeling genes in biliary and pancreatic cancers: ARIDIA, PBRMI, and $B A P 1$. Wang and colleagues provide an indepth assessment of the Xp11 translocation in renal cell carcinoma and the mesenchymal counterparts (Xp11 neoplasm with melanocytic differentiation and alveolar soft part sarcoma), with novel insights into the clinicopathologic features, prognosis, treatment, and classification. The Xp11 translocation mesenchymal neoplasms may represent a distinct entity that shares some molecular features with the Xp11 translocation renal cell carcinoma and broadens the spectrum of Xp11 translocation-associated neoplasms.

The role of Eph/ephrin and its contributions to the tumor microenvironment and potential targeted therapies are explored by Zhou and colleagues. The knowledge of specific molecular features is central to the therapeutic planning for some tumor types. The treatment of thymic epithelial tumors is discussed for molecular targeted therapy by $\mathrm{Du}$ et al. Sun et al. highlight the importance of oncogenic signaling pathways and pathway-based therapeutic biomarkers in lymphoid malignancies. Finally, Reyes-Barron et al. focus on the next-generation sequencing for minimal residual disease surveillance in acute lymphoblastic leukemia.

This Special Issue highlights recently identified molecular targets and pathways and provides a critical evaluation of their diagnostic and therapeutic roles in the management of cancer patients. The Guest Editors wish to express their gratitude to the authors who have contributed to this special issue. 
Guest Editors:

Liang Cheng

Departments of Pathology and Urology

Indiana University School of Medicine

Indianapolis, IN, USA

Qiu Rao

Department of Pathology

Nanjing Jinling Hospital and Jinling Clinical Medical College

Nanjing University School of Medicine

Nanjing, Jiangsu, China

Xiao-Tong Wang

Department of Pathology

Nanjing Jinling Hospital and Jinling Clinical Medical College

Nanjing University School of Medicine

Nanjing, Jiangsu, China
Bo Zhang

Department of Pathology

Health Science Center of Peking University

Beijing, China

Rodolfo Montironi

Institute of Pathological Anatomy and Histopathology

Polytechnic University of the Marche Region (Ancona) and United Hospitals

Ancona, Italy 